\title{
An Access of Smart grid Standards and its challenges
}

\author{
Vijayendra V K, Pavan M N, Neethi M
}

\begin{abstract}
Standards are the set of details of a product that defines the applicability of a product for a distinct usage or details that determines the fulfillment of a system or a device for a given application. The development of smart grid over past few decades had led to development of various standards in various areas pertaining to smart grid deployment. These standards are already well established or being under developmental stage covering vast fields of power system elements and variety of functionalities. An overview of standards that are deployed in the smart grid development environment mainly concentrating on Energy Management System (EMS) \& SCADA, substation automation, metering, Electric Vehicles(EVs), Distributed Generation (DG), internet related and cyber security is presented in this paper.
\end{abstract}

Keywords - Smart grid, SDOs, standards, interoperability.

\section{INTRODUCTION}

The electricity demand world-over is estimated to double between 2000 and 2030, with an average growth rate of 2.4\%, which is faster than the estimation for any non-renewable energy resource [2]. With the increased demand on one-side, there is focus on various initiatives to decrease the carbon footprints since there is increase in percentage of renewable energy based generation in overall energy mix. With this increased demand on one side and increased penetration of renewable energy based generation on other side, smart grid has a predominant role in dealing with energy and environmental challenges. Ageing infrastructure of existing conventional grid, fast growth of global energy demand, global environmental concerns, increased focus on electrification of transportation and consumer awareness are some of the major drivers for transformation from conventional grid to smart grid. We can define smart grid in simple terms as "The power system from production to consumption which is fully integrated with communication technology at all stages" [2]. Smart grid can also be defined as an cyber-enabled, end-to-end power system, from source of generation, along the transmission and distribution systems till end consumer that will 1) Allow for interconnection of sporadic renewable energy sources and helps in de-carbonizing power systems, 2) Allow for secure and reliable two-way power and information flows with security 3) Enable customers to make choice by ensuring

Revised Version Manuscript Received on 10 September, 2019.

Vijayendra V K, PG Student, Energy Systems \&Managament, JSS S\&T University, Mysuru, Karnataka, India.

(Email: vijayendravk@gmail.com)

Pavan M N, PG Student, Energy Systems \&Managament, JSS S\&T University,Mysuru, Karnataka, India.

(Email: pavankptcl@gmail.com)

Neethi M, Asst Professor, Department of E\&E Engg., JSS S\&T University, Mysuru, , Karnataka, India.

(Email: neethisjce@gmail.com) energy efficiency and effective demand management 4) Capability of self-healing in case of any abnormal system operations leading to disturbances 5) Provide resilient system operations against physical and cyber-attacks by hackers [2].

Various applications, devices, technological solutions and standards/protocols have been developed for smart grid systems or are still in the developmental stages. Major challenge for development of smart grid solutions is that smart grid as a system is lacking standards which are widely accepted world-over. This is an obstacle for further development and integration of latest smart devices, smart meters and renewable energy based generation.

Some of the important objectives that can be realized with standardization efforts in smart grid environment are easy interoperability, secured information flows, enhanced safety of latest products and systems suitable for smart grid environment and compact set of protocols. The critical requirement for making the smart grid system a reality is adoption of interoperability standards for the overall system [3]. With increase in number of smart devices and application day by day, well established standards are very much required for ensuring interoperability, integration and effective communication of various devices in the smart grid environment.

There are several attempts from various regional-level, national-level and international-level organizations for achieving smart grid standardization objectives and goals. These organizations are referred to as SDOs (Smart grid Development Organization).

Earlier works and publications focus mainly on standards that are relevant to a particular domain of interest. However, works which provide an overview of standards in smart grid environment are limited. Thus, an attempt is made to provide an overview of major standards related to SCADA \& EMS, Metering, Substation automation, Electric vehicles, Cyber security and Distributed Energy Resources (DERs), which are under developmental stage and considered to be a future potential for the grid.

\section{EVOLUTION OF THE GRID}

During earlier days, generation was confined to large power generating stations only as shown in Figure 1. But in the present day situation, sources of generation being distributed at various points in the power system, power generating sources can be located throughout the transmission and distribution network. In the past, power 
flow was in one direction, i.e., from generating stations to theend consumers through the transmission and distribution network. Sometimes, few large industrial or commercial customers with their own co-generation plants were able to interconnect their own co-generation facilities to the grid network. This interconnection of small scale generation proved to be costly and time consuming since it involved engineering to meet requirements which are different from utility to utility as well as different regulatory requirements.
Safety conditions, electrical interconnection rules were not common among power sector entities. The various components required for the grid interconnections differed based on specifications by vendors for the generation and transmission equipments, control \& communications equipments which necessitated special interfaces. Gradually, standards were formulated to provide a common platform for grid interconnections [5].

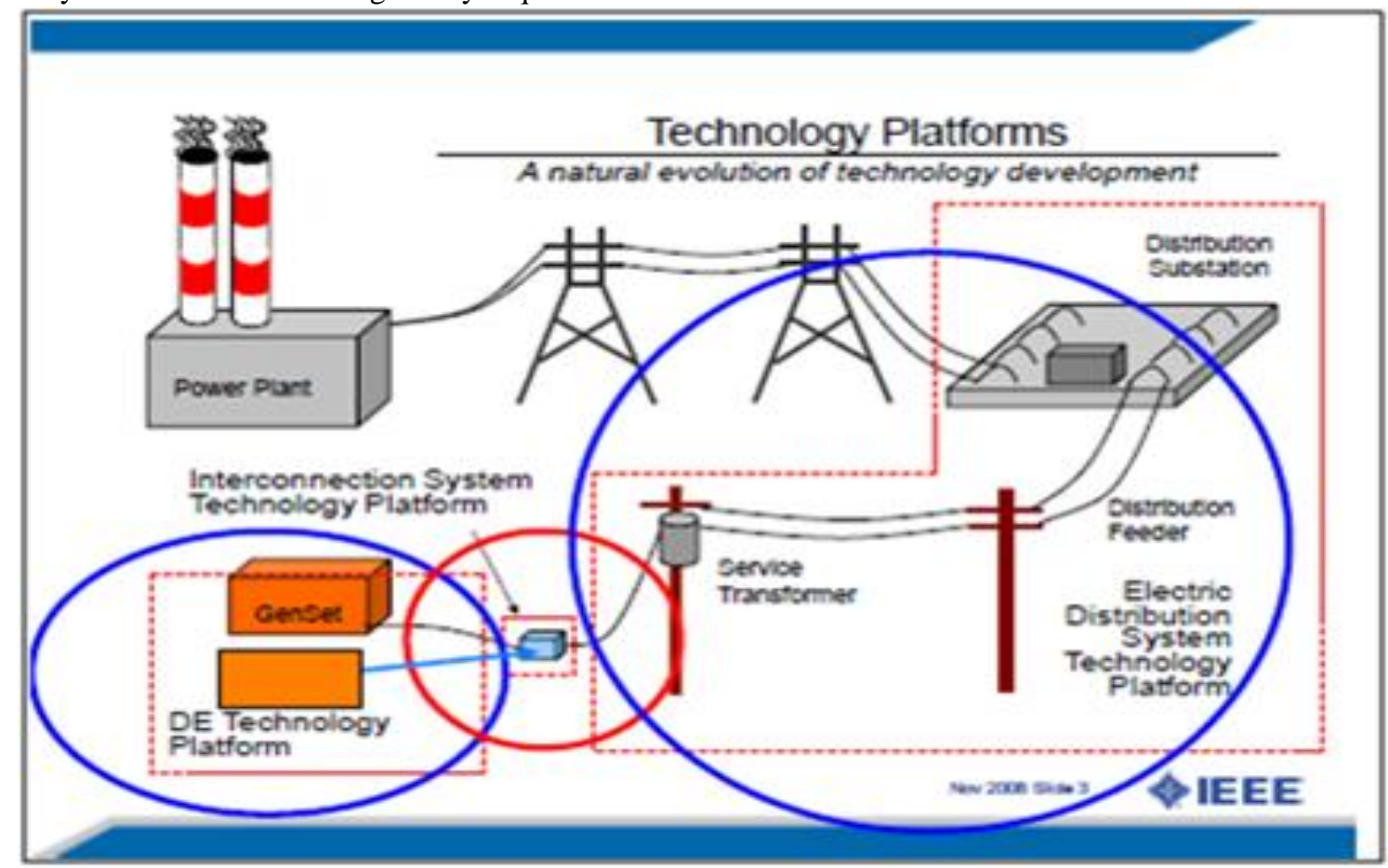

Figure 1: Conventional/Traditional power system

\section{A. Conventional grid Vs Smart grid:}

The traditional grid also called as conventional power grid is radial interconnection of different power systems components namely, synchronous generators, generator transformers, power transformers, transmission lines \& sub-stations, distribution lines \& sub-stations and different categories of consumer loads. These infrastructures are situated away from the load centers and electric power is transmitted through a network of lengthy transmission and distribution lines.

In order to overcome the drawbacks of this traditional grid, smart grid provides a secure and dependable option for service. It provides for a bi-directional communication between the utilities and the consumers. The smart grid is the new intelligent grid which is capable of monitoring working of the interconnected grid, priority of power utilization by various consumers and provides real-time information regarding the various events in the grid. Smart substations, consumers equipped with smart meters and smart appliances/devices advanced synchrophasor technologies constitute few important components of smart grid. The smart grid is the next-generation intelligent grid that is provided with most advanced products and communication technologies to ensure more efficient operation.

To efficiently handle high penetration of renewable energy based generation into the grid network, smart grid is the only system which could be able to provide solution to handle the complexities of the future grid.

\section{B. Benefits of smart grid:}

Important advantages of deployment of smart grid are reliable \& uninterrupted power supply for all the consumers, reduction in transmission \& distribution losses, capability to handle increased penetration of renewable energy based generation, cyber secured electrical grid network, energy storage opportunity on a large scale to support micro grids, flexibility with multiple options to allow for consumer interaction with electricity market, dynamic pricing of energy based on power exchange rates and demand side management options for utilities [5] .

The major achievements in the path of smart grid implementation are country wise policies and planning committees for smart grid deployment, successful trial project implementations in selected cities, development of few standards for smart grid interoperability, promoting the usage of distributed renewable energy based generation and research conducted to analyze the cost effectiveness of new technologies for deployment of smart grid.

\section{Smart grid standards:}

Standards are the set of details of a product that defines the applicability of a product for a distinct usage or details that determines the fulfillment of a system or a device for a given application. 
Progress made in development and deployment of smart grid has guided towards to evolution of diverse standards and interoperability in power system. These standards are aimed at covering a variety of elements and functionalities in a power system.

\section{NEED FOR SMART GRID STANDARDS}

\section{A. Relationship among smart grid stakeholders:}

The main requirement for Smart grid is to maintain a balance between energy supply and demand through real-time monitoring which is made possible by using bi-directional Inter Connecting Transformers (ICT) [6]. The cost of generation of electricity depends on several factors including quantum of power being handled, the methodology adopted for power generation, location of power plant and consumption time. Accordingly, the electricity pricing changes due to variation in consumer consumption patterns. Thus, it is important for both utilities and consumers to exchange information related to power supply and requirements at different periods of a day with each other. Further, it is critical to ensure reliability not only power generation, transmission and storage systems but also the reliability of the information exchanged between utilities and customers. For instance, it may be possible that many of the consumers use email, mobile apps, or web-based dashboards through a system or mobile phone for monitoring the energy usage at home or at an industry. This will lead to effective energy management. However, for such kind of energy management, transmitting critical data pertaining to energy usage and consumer privacy in the internet environment cannot meet the security and latency requirements of power systems. For ensuring reliable smart grid operations, cyber security technologies in particular will have a major role to play.

The major challenge for achieving the next generation smart grid lies in the gap between existing standards and requirement of electricity market and lack of interoperability among few available standards. Thus, in order to bridge this gap between existing few available standards and future requirements, standardization process of the Smart Grid has been promoted by various Standards Developing Organizations (SDOs). The important role of standards for the Smart Grid development has already been achieved world over by various government bodies and industrial organizations. These organizations recommend the development and deployment of standards to ensure that investments made in the Smart Grid today will be worthy even in the future. This standardization vision is to ensure different products from different vendors to interoperate seamlessly, to speed up the innovations, to assist consumers to make choices to choose the products, to create economies so as to bring down overall costs and to open up smart grid markets worldwide.

The International Electro-technical Commission (IEC), one of the major international SDO for the Smart Grid has specified that a higher level of syntactic and semantic interoperability is necessary for different products, technologies, solutions, and systems which constitute the smart grid related systems. protocols for accomplishing devices and equipment

Other than SDOs, there are also many technical forums, consortia and panels, which are actively involved in process of promotion of the smart grid standardization.

With an increase in deployment of smart products and applications at the consumer end, standards and protocols have become extremely important requirement for achieving seamless integration of a large number of such products and systems into the smart grid environment and enabling communication and exchange of information between them.

The inter-relationship among the SDOs, utilities, vendors and other user groups can be represented as shown in figure-2:

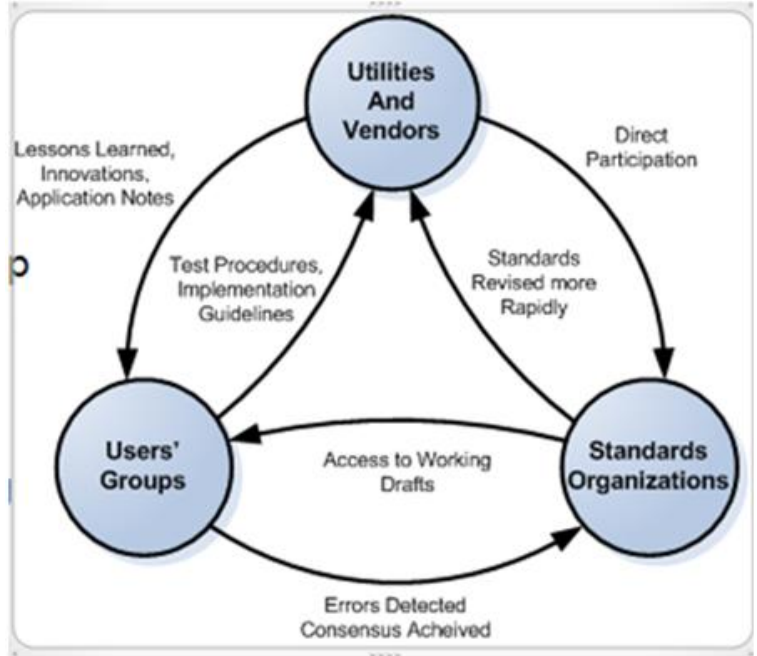

Figure 2: Relationship among SDOs, Utility, Vendors and user group in developing smart grid standards

\section{B. Smart grid related organization (SDO):}

SDOs are those organizations that are responsible for development, time to time revision, co-ordination among entities, and amendment of technical standards related to smart grid. These organizations deal with various sets of standards that address a given set of requirement. They also deal with specifications for the identified applications that result in acceptance as formal standards which are approved by competent authority. Some of these developed standards may also be informal as they are not approved by law but adopted by industries voluntarily depending on requirements.

Besides these SDOs, there are several other technical forums, consortia, panels, regulatory and marketing organizations which are also actively working in cooperation with the SDOs for development/evaluation/review of smart grid-related technical issues and in promoting the process of standardization.

Classification of these SDOs is based on their functions, positions, and application domains. SDOs can be at local-level, regional-level, or international-level, and might be governmental, semi-governmental, or non-governmental entities [6].

Few important smart grid related SDOs are International Electro-technical Commission (IEC), Institute of Electrical and Electronic Engineers (IEEE), National Institute of Standards and Technology (NIST), International Organization for Standardization (ISO), International 
Telecommunication Union (ITU), Society of Automotive Engineers (SAE) International Internet Engineering Task Force (IETF), European Committee for Standardization (CEN), European Committee for Electro-technical Standardization (CENELEC), Telecommunications Industry Association (TIA), Alliance for Telecommunications Industry Solutions (ATIS).

Other major smart grid forums and technical consortia and panels associated with smart grid are Wifi alliance, Zigbee alliance, WiMAX forum, UCA international user group, Organization for the Advancement of Structured Information Standards (OASIS), National Electrical Manufacturer Association (NEMA), Home Grid Forum, GridWise Architecture Council (GWAC) etc.

\section{C.NIST Smart grid framework:}

Smart grid framework release 1.0 by NIST was published during January 2010. This was an important milestone in development of standards for smart grid. This framework documented the progress made in development of standards at that time. The NIST framework release 1.0 described a high-level conceptual reference model for the smart grid. Framework identified about 37 smart grid related standards published in the Catalog of Standards $(\mathrm{CoS})$, and an further additional 61 standards were identified for further review and comments by the panel.

The publication was updated with NIST Smart Grid framework and roadmap for Interoperability Standards (Release 2.0), in February 2012 and Release 3.0 in May 2014 [10].

\section{IMPORTANT STANDARDS RELATED TO SMART GRID}

Most commonly used standards related to smart grid can broadly be identified under the domain of SCADA \& EMS, Metering, Substation, EVs, Distributed Generation, Internet related and Cyber security as presented in figure 3 :

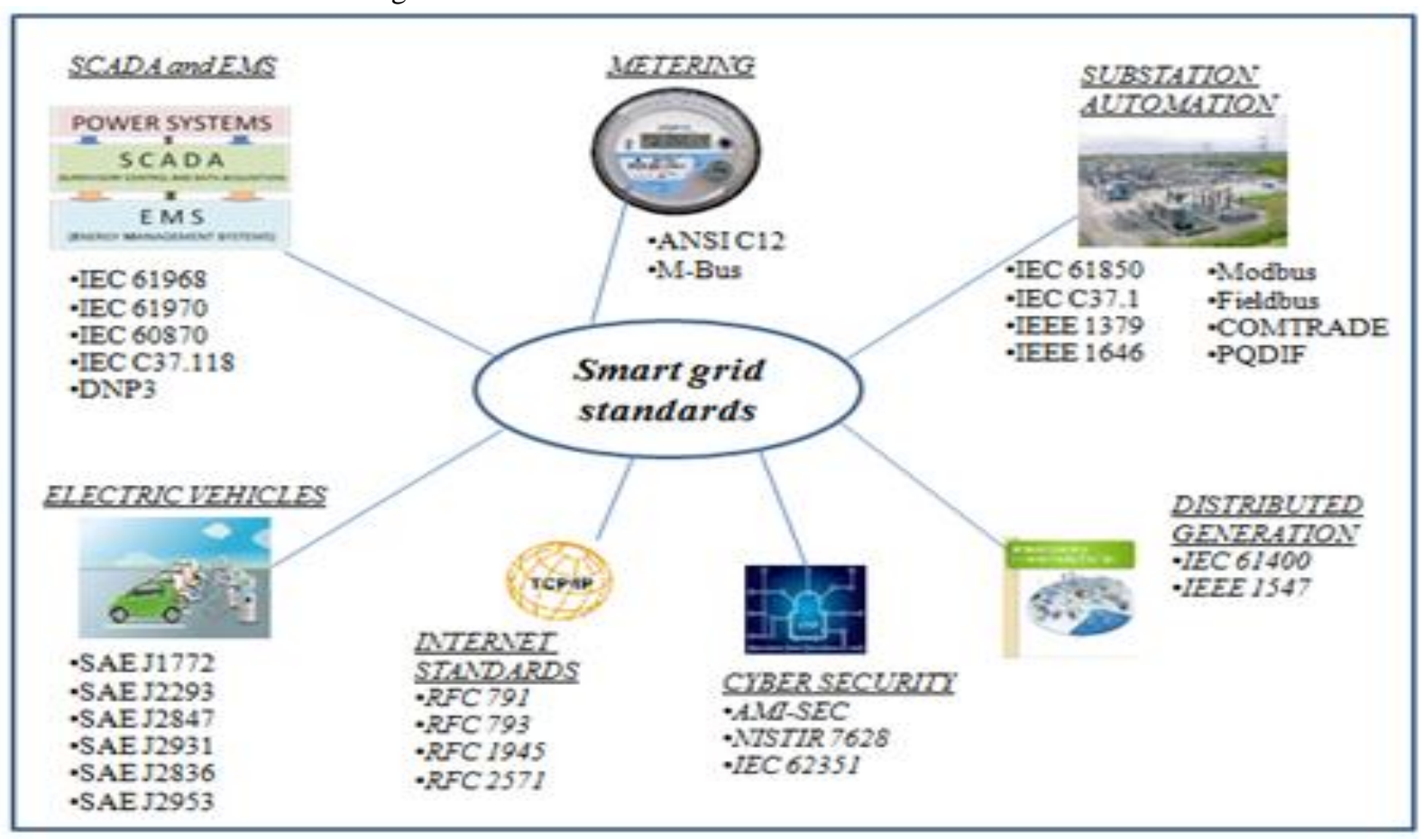

Figure 3: Commonly used smart grid related standards.

Brief descriptions about the important standards are as below:

\section{A. SCADA \& EMS:}

1. IEC 61968: This is known as Distribution energy management standards. It is used for Advanced Metering Infrastructure (AMI) back office interfaces. This standard works on various operating systems, various programming languages and suitable for various database schema systems.

2. IEC 61970:. This is also known as Energy Management System Application Program Interface (EMS-API) [7]. This protocol defines a semantic information model in the area of power transmission. IEC 61970 also provides an abstract Application Program Interface (API) for data exchange which is technology and platform independent.

3. IEC 60870: This is an Inter-Control Center Protocol (ICCP) which specifies systems used in power system applications for Supervisory Control and Data Acquisition (SCADA). This provides tools and interfaces for SCADA systems. This lacks the security aspects such as authentication or encryption.

4. IEEE C37.118: This is a standard used for synchrophasors which defines requirements for phasor measurement units (PMU) used in transmission systems. It also defines suitable communication protocols for exchange of phasor data in transmission elements. IEEE C37.118 is developed for publishing synchronized phasor measurement data. This specifies methods for quantification of phasor measurement data and testing methodology in order to ensure that standard format is maintained.

4. DNP3: This is normally deployed in transmission substations for control and monitoring of various equipments. This is deployed in SCADA systems to specify communication protocols among SCADA master station,

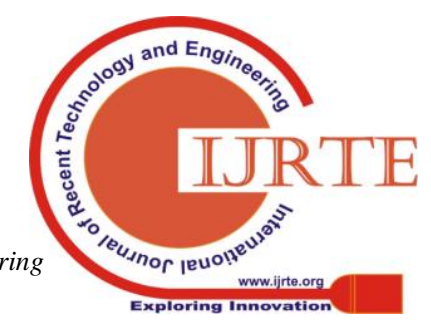


Remote Terminal Units (RTUs) and Intelligent Electronic Devices (IEDs).

\section{B. METERING:}

1. ANSI C12: ANSI C12.19 standard provided metering tables which is internal to the meter. ANSI C12.22 provided communication specifications for metering tables.

Protocol for metering applications is defined by ANSI C12 suite (e.g., ANSI C12.18, 12.19, 12.20, 12.21, 12.22). This suite specifies requirements and specifications for optical ports, electric meter accuracy classes, end device data tables, specification for modem and networks interfacing.

2. M-BUS: This is also known as Meter-Bus. This standard is designed mainly for low cost, battery power devices. This is widely adopted for remote electricity and gas utility meter readings.

\section{SUBSTATION AUTOMATION:}

1. IEC 61850: This deals with substation automation systems, distributed renewable energy based generation (solar PV, wind energy, fuel cells etc) and distribution automation systems.

This protocol specifies communication systems in substations with focus on interoperability among intelligent electronic devices (IEDs). This enables the functions such as protection, monitoring, control and automation in substations. In order to have differentiation among different applications and prioritize data flows, this protocol defines five different types of services for service interface, substation events and time synchronization.

2. IEEE C37.1: This protocol defines system architectures and functions in a substation including Human Machine Interfaces (HMI), protocol selections and matters related to implementation. It also specifies network performance issues and specifications related to availability, expandability, maintainability, reliability and security.

3. IEEE 1359: This provides set of implementation and practicing guidelines in an transmission substation for communications and interoperation of IEDs and RTUs. This protocol helps in eliminating the requirement for slow and cost ineffective methods for interfacing equipment to other equipment within a substation.

4. IEEE 1646: This specifies the data travel and delivery within and outside to substation protection system, controls system and data acquisition system. This defines delay times between processes running on two different end to end times.

5. Modbus: This is used for connecting a SCADA master station unit with RTUs. It has a master-slave model based messaging pattern. It supports serial (two transmission modes: ASCII and RTU), as well as Ethernet (TCP/IP) open serial communications protocol.

6. Fieldbus: Fieldbus also called as IEC 61158, It is mainly used as for computer networks in industrial systems. This is used for real-time distributed control. This protocol is used at the bottom of the control chain that links PLCs to components deployed at field such as sensors, actuators and electrical motor drives. times for communication messages among the equipments systems. This includes both processing and transmission protocols. In substation automation systems, it is used as an

7. COMTRADE: IEEE 37.111 is also called as COMTRADE (Common format for Transient Data Exchange for power systems). This protocol defines a file format for storing various electrical parameters viz., current, voltage, power, frequency, etc., recorded by IEDs at the time of disturbance or abnormal operating conditions in a power system. At the time of such power system disturbances, COMTRADE files can be obtained from various transmission substations and those files can used to analyze the power system disturbance events. This helps in understanding causes for disturbance and to arrive at methodology for mitigation of such events in future. The file format for such disturbances in power systems has been standardized under C37.111 or COMTRADE.

8. PQDIF: IEEE 1159.3 is also known as PQDIF. This is also a standard binary file format for exchange of power quality related measurements and simulation data such as voltage harmonics, current harmonics and power measurements. To standardize data formats from different simulation studies, measurements and analysis tools from many vendors, this standard was introduced jointly by IEEE and EPRI. PQDIF is similar to COMTRADE in structure, but it is used mainly to provide power quality related data.

\section{DISTRIBUTED GENERATION:}

1. IEC 61400: This standard provides design specification and data exchange patterns for wind power plants control and monitoring. It allows for exchange of information between a wind power plants and control center. This provides set of requirements which is not dependent of type and make of wind turbines. This also specifies a set of design specifications to ensure robustness in wind turbine designs from different manufacturers. This includes design specifications for all type of wind turbines including turbine gearboxes, methods for acoustic noise measurement, performance testing, rotor blades testing, power quality measurements of grid connected wind plants, and protection against lightening [7].

2. IEEE 1547: This protocol provides specifications for interconnection of distributed energy resources with the grid network. This provides guidelines for physical and electrical interconnection and interoperability of distributed energy resources with power systems. This provides requirements for performance, operation, testing and safety.

\section{E. ELECTRIC VEHICLES:}

Standards related to Electric Vehicles and their charging is developed by SAE which is a global society for automotive vehicle industry.

1. SAE J1772: This standard specifies various methods for charging and connector requirements for level 1, level 2 and DC chargers [7].

2. SAE J2293: This provides requirements for Electric Vehicles (EVs) and the off-board electric vehicle supply equipment (EVSE). It provides functional specifications, network architectures and communication specifications for transfer of electrical energy to an EV from an electric utility in North America [7].

Published By: 
3. SAE J2836: This provides set of use cases for communications between plug-in electric vehicles (PEV) and the grid network, between customers and PEV and between PEV \& off-board DC chargers. It also specifies specifications for PEV communicating ad DER which will be used as a storage system in such a scenario [7].

4. SAE J2847: This provides specification and requirements for communications of PEV using Smart Energy Profiles, between PEV and off-board DC charger, for PEV as a DER, between PEV and their consumers, and between wireless charged vehicles and wireless EV chargers [7].

5. SAE J2931: This provides the specifications for digital communication between utilities and PEV/EVSE, Energy Services Interface (ESI), AMI and Home Area Network (HAN) [7].

6. SAE J2953: This standard deals with the issues of interoperability of PEV and EVSE. It provides set of specifications by which a particular PEV and EVSE pair can be considered as interoperable in different platforms. Also, it provides testing guidelines to ensure the interoperability of PEV and EVSE from different manufacturers. SAE J2953 has three tiers of interoperability testing, namely mechanical, charge and safety feature functionality, indefinite \& dynamic grid events andampacity control [7].

\section{F. CYBER SECURITY:}

1. AMI System Security Requirements (AMI-SEC): This is developed by AMI-SEC task force. This provides a set of security specifications for AMI, which advantage of the utilities and vendors. It provides specifications related to security issues for procurement of smart meters.

2. NISTIR 7628: 7628 provides the guidelines for cyber security related issues in smart grid environment. This provides set of specifications in three volumes: Vol. 1 strategy for smart grid cyber security, architecture, Vol. 2 smart grid privacy and Vol. 3 - supportive analyses and references. NISTIR 7628 provides a framework for development of effective strategies for cyber security. It specifies the guidelines for assessment of risks involved in cyber security and helps in identifying suitable requirements for cyber security.

3. IEC 62351: This defines requirements for cyber security for power systems management and data exchange associated with it. IEC 62351-1 specifies various aspects of networking and system security related with operation of power systems. IEC 62351-2 defines terms and acronyms used in the standards. IEC 62351-3 to IEC 62351-6 specifies messages, procedures and algorithms for different applications. IEC 62351-7 to IEC 62351-11 addresses data security, access control, key management and others.

\section{G. INTERNET STANDARDS:}

Internet related protocols are developed by Internet Engineering Task Force (IETF). Protocols are released in the form of RFC (Request for Comments) for review and for accepting comments from all the stakeholders. Few important protocols are:

1. RFC 791: Provides the framework related to Internet Protocol (IP).

2. RFC 793: Provides the framework related to Transport
Control Protocol (TCP).

3. RFC 1945: Provides the framework related to Hyper Text Transfer Protocol (HTTP).

4. RFC 2571: Provides the framework related to Simple Network Management Protocol (SNMP).

\section{CHALLENGES IN IMPLEMENTATION OF SMART GRID\& RESULTS}

In order to transform the present dumb grid network into a smarter grid, numerous smart technologies, devices have been under development and few of them have been deployed as well. Interoperability among various smart devices and different platforms is a major factor that is very important during design of smart grid. Even though lot of progress has been made for development and deployment of smart grid at different levels, many challenges still remains to be addressed. Especially, the domain of smart grid related standards and protocols require lots of attention. Few of the challenges include: lack of awareness of existing standards in implementation of smart grid among utilities \& designers, interoperability among the devices and various available platforms, complexity involved in the smart grid implementation and technical challenges involved with variety of power system elements in the grid network.

\section{CONCLUSION}

Design, development and deployment of smart grid are of great importance for meeting the ever increasing demand for electricity. Deployment of smart grid is a continuous and a learning process for which standards plays major role providing a common platform. A number of SDOs and other organizations are constantly working on smart grid related standards to provide concrete set of guidelines for successful implementation of smart grid. An attempt has been made in this paper to provide an overview of important smart grid related standards. Once initially developed, these standards need to be reviewed and revised periodically based on the practical use case experiences and feedback from implementation of smart grid. As a continuous learning process, standardization plays an important role to build a secure, reliable, advanced and smart electric power grid which is capable of communicating both ways, interoperability among various devices and control capabilities.

\section{REFERENCES}

1. JamesMomoh, Smart grid - Fundamentals of Design and Analysis, WILEY, 2016.

2. IEEE Vision for Smart Grid Controls: 2030 \& Beyond.

3. Dr. Yuri Khersonsky, "Don't re-invent the Smart Grid; use IEEE Standards to make it Smart".

4. Richard DeBlasio\& Cherry Tom, "Standards for the Smart Grid”, IEEE Energy 2030, Atlanta, Georgia, USA, 2008.

5. C. P. Vineetha* and C.A. Babu, "Smart grid Challenges, Issues and Solutions". 
6. Takuro Sato Daniel M. Kammen Bin Duan Martin MacuhaZhenyu Zhou Jun Wu Muhammad Tariq Solomon AbebeAsfaw, Smart Grid Standards: Specifications, Requirements, and Technologies", WILEY, 2015.

7. M. Kuzlu, "A Comprehensive Review of Smart Grid Related Standards and Protocols", ICSG Istanbul 2017.

8. Final report of the CEN/CENELEC/ETSI Joint Working Group on Standards for Smart Grids.

9. NIST Framework and Roadmap for Smart Grid Interoperability Standards, Release 3.0. 